This item was submitted to Loughborough's Research Repository by the author.

Items in Figshare are protected by copyright, with all rights reserved, unless otherwise indicated.

\title{
Cooperative multi-hop wireless network with robustness to asynchronism
}

PLEASE CITE THE PUBLISHED VERSION

http://dx.doi.org/10.1109/EW.2010.5483499

PUBLISHER

(c) IEEE

VERSION

VoR (Version of Record)

LICENCE

CC BY-NC-ND 4.0

REPOSITORY RECORD

Elazreg, A.M., Shakiru K. Kassim, and Jonathon Chambers. 2019. "Cooperative Multi-hop Wireless Network with Robustness to Asynchronism". figshare. https://hdl.handle.net/2134/6856. 
This item was submitted to Loughborough's Institutional Repository (https://dspace.lboro.ac.uk/) by the author and is made available under the following Creative Commons Licence conditions.

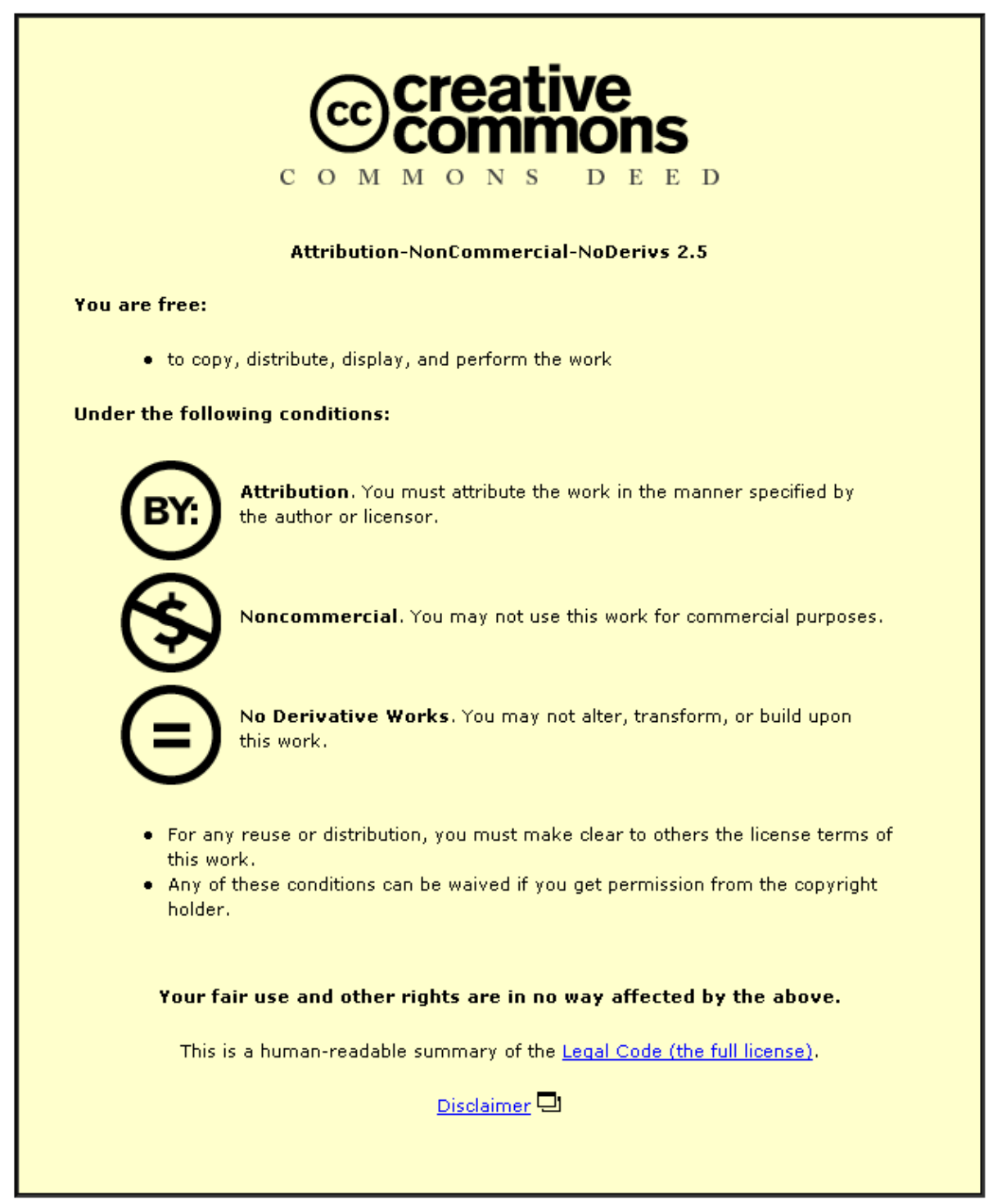

For the full text of this licence, please go to: http://creativecommons.org/licenses/by-nc-nd/2.5/ 


\title{
COOPERATIVE MULTI-HOP WIRELESS NETWORK WITH ROBUSTNESS TO ASYNCHRONISM
}

\author{
A. M.Elazreg, S. K.Kassim and J. A.Chambers \\ Advanced Signal Processing Group, Electronic and Electrical Engineering Department, \\ Loughborough University, Leicestershire, UK,LE11 3TU \\ Email:\{A.Elazreg, S.K.Kassim, J.A.Chambers\}@lboro.ac.uk
}

\begin{abstract}
We investigate the use of a timing synchronization technique within cooperative multi-hop networks with and without direct transmission between the source and destination nodes. In particular, we employ a parallel interference cancellation (PIC) technique to combat the effect of timing misalignment of the received signal at both the relay and the destination nodes. We compare the advantage in using direct transmission (DT) to assist in reducing the end-to-end bit-error-rate (BER) at the receiver with using multiple level cooperation among all nodes of each hop without direct transmission. Using 8-PSK constellation signals, our simulation results show that increased cooperation among neighboring nodes delivers improved end-to-end BER as compared to direct transmission assisted cooperation.
\end{abstract}

Index Terms - Multiple-input multiple-output (MIMO), cooperative diversity, virtual antenna arrays (VAAs), distributed spacetime block code (DSTBC), parallel interference cancelation (PIC).

\section{INTRODUCTION}

It has been shown in the literature that utilizing multiple-input multiple-output (MIMO) systems can improve the reliability of wireless communication by utilizing diversity gain whilst preserving full data rate [1]. However, due to cost constraints, size limitation and hardware complexity, it is usually difficult to pack multiple antennas in one mobile communication node (or other wireless device) whilst ensuring spatially uncorrelated channels between the transmitter and receiver, a condition necessary to exploit fully the potential gains of (MIMO) systems.

To overcome this problem, the concept of cooperative communications [2] has recently gained much interest due to its potential ability to realize the performance gains of (MIMO) wireless systems without multiple antennas at individual nodes. In cooperative communications systems, mobile nodes and other wireless devices can share their information and transmit cooperatively as a virtual antenna array (VAA) [3], thus providing spatial diversity. In order to maximise the diversity advantage in cooperative systems, the deployment of a "conventional" orthogonal space-time block code (STBC) has been suggested in a distributed fashion [2] [4] [5] (sometimes called a distributed space time block code (D-STBC)) using two single antenna cooperating relay nodes. In [6] and [7] it was shown that the diversity orders offered by orthogonal STBC (O-STBC), quasi-OSTBC (QO-STBC) and extended orthogonal STBC (EO-STBC) in pointto-point (MIMO) systems are achievable over a two-hop network when these codes are deployed across cooperating relay nodes. Much existing research on D-STBC strategies assumes perfect synchronization among cooperative users at the symbol level, which means all transmitted symbols from all nodes are assumed to arrive at their destination node at the same time. Practically, this is not a true representation of the real transmission environment. The assumption of accurate symbol level synchronization in wireless networks (such as ad hoc networks), is difficult or even impossible to achieve owing to the signalling overhead [8]. This lack of synchronization results in intersymbol interference (ISI) between the received symbols from the relays at the destination, thus destroying the orthogonality of the STBC. Recent attempts at limiting or eliminating this ISI have been reported in [8] [9], where an equalization technique is employed at the destination to reduce the effect of asynchronous receive signals, however, this technique incurs increased receiver complexity. Some other techniques were also used to overcome the problem of signal synchronization, which is a simpler approach based on parallel interference cancellation (PIC) techniques, used in CDMA systems [10] has been proposed in [11]. The authors demonstrated that for a single antenna source, relays and destination nodes, the use of the PIC technique, at the destination, has the potential of delivering diversity order close to the number of cooperating relays, provided the signal transmitted directly from source to destination is appropriately combined with the signals transmitted from the relay nodes, thus increasing the receiver signal-to-noise ratio (SNR). However, due to potentially high pathloss between the source and destination, the direct transmitted signal suffers large degradation in energy [12], thus making its promised enhancement in the receiver SNR unrealistic.

Since the essence of relaying is to reduce the effect of pathloss on the data transmitted from source to destination [13], in this work we propose a new relaying solution that employs PIC detection at both the relay and destination nodes, without direct transmission from the source to destination, thereby promising to deliver diversity order close to the multiplication of number of cooperating nodes at the relaying stage and the destination. For comparison purpose, we show the end-to-end BER performance of our proposed scheme without direct transmission and the BER performance of two stages with direct transmission as shown [11].

The paper is organized as follows, Section II describes a generalized cooperative multi-hop wireless network. Cooperative relaying with D-STBC under imperfect synchronization is presented in Section III. In the following Section IV, conventional STBC detection 
is introduced. To mitigate the interference at the symbol level at the destination nodes, the full (PIC) detection scheme is introduced in Section V. The simulation results are given in Section VI. Finally, in Section VII, conclusions are drawn.

In the remaining part of this paper, [.] $]^{T},[.]^{*},||,. \Re\{$.$\} and \{.\}^{H}$ denote transpose, conjugate, absolute value, real part of a complex number and Hermitian (complex conjugate transpose) operations , respectively. $C N\left(0, \sigma^{2}\right)$ represents a Gaussian distributed complex random variable with the standard variance of $\sigma^{2}$ (i.e. $0.5 \sigma^{2}$ per dimension).

\section{SYSTEM MODEL}

In this section, we describe the system model for our proposed multihop wireless network with cooperative relaying. As shown in Fig. 1, the source node transmits information with the cooperation of adjacent mobile terminals (AMTs), to the destination node via cooperative relay nodes. The destination node together with its AMTs receive the information which is jointly decoded and the required message extracted by the destination node. The level of cooperation at each hop depends on the numbers of "idle" terminals that ensure error free communication during cooperation, for example nodes within a Bluetooth range [14] can be employed.

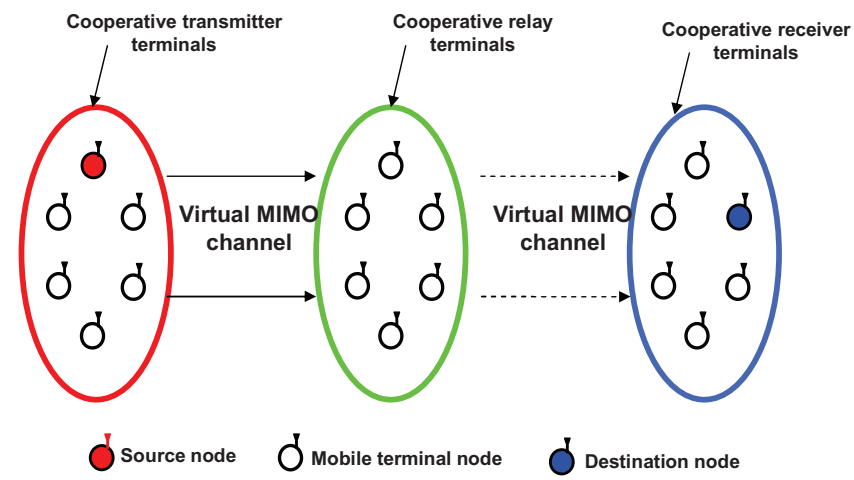

Fig. 1. Cooperative communication in a multi-hop wireless network.

In this model, we assume that all links are modeled as Rayleigh fading frequency flat channels (except the cooperative link). We also assume that the channel coefficients are unchanged during the transmission of a signal code block (quasi-static) and all terminal nodes are equipped with a single antenna and are assumed to operate in half duplex mode. In the next section we describe the level of cooperation at each hop and discuss the implementation of the D-STBC under imperfect synchronization.

\section{COOPERATIVE RELAYING WITH D-STBC UNDER IMPERFECT SYNCHRONIZATION}

In this section the example of 2-hop communication is described with cooperative relaying with distributed space time block coding [1] in both hops as shown in Fig. 2. This model has an information source cooperating with one of its AMT nodes, two relay nodes cooperate with each other and a destination node also cooperates with one of its AMT nodes. As in most cooperative communication systems, there are two phases involved.

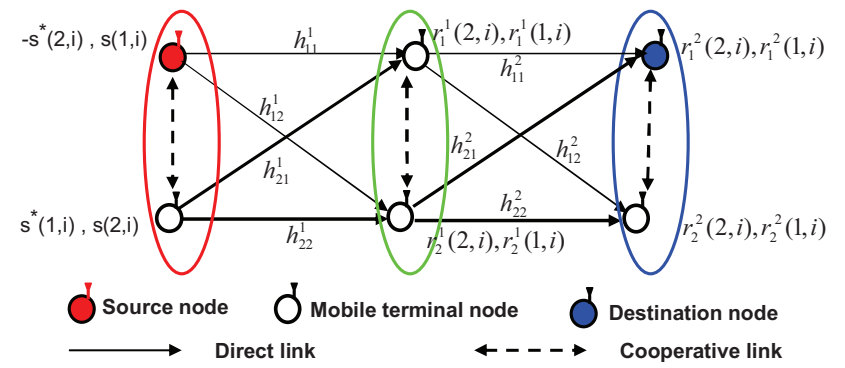

Fig. 2. Basic structure of a 2-hop communication model with cooperative transmission.

Phase one Broadcast: The source node cooperates with one of its AMTs to encode its symbols $\mathbf{s}(i)$, which are grouped into pairs $s(1, i)$ and $s(2, i)$ within the distributed space time block coding technique and then it transmits codeword $S^{1}$ as shown in equation (1) while relay nodes $R_{1}$ and $R_{2}$ receive the signal and prepare for the STBC operation in phase 2. At a given time slot two signals are simultaneously transmitted from the source node and one of its AMTs. The signal transmitted from the source node is denoted by $s(1, i)$ and from AMT by $s(2, i)$ in the first time slot. During the next time slot signal $-s^{*}(2, i)$ is transmitted from the source node and signal $s^{*}(1, i)$ is transmitted from the AMT.

$$
S^{1}=\left[\begin{array}{cl}
s(1, i) & s(2, i) \\
-s^{*}(2, i) & s^{*}(1, i)
\end{array}\right]
$$

Due to imperfect synchronization resulting from different propagation delay in both hops, the transmitted signal in the first hop will not arrive at node $R_{j}, j=1,2$ at the same time due to time delay between source node and its AMT node, similarly in the second hop the received signal will not arrive at the destination node and its AMT node at the same time due to time delay between $R_{1}$ and $R_{2}$, which means exact synchronization is difficult or impossible in both hops [15]. As shown in Fig. 3 there is normally a timing misalignment of $\tau_{j}^{k}$, among the received versions of the signals where $k=1,2$ is the number of hops. At this point we assume that $\tau_{j}^{k}$ is smaller than the sample period $T$ in both hops as shown in Fig. 3, this assumption is easy to meet in practice [11], such relative time delay will cause ISI from neighboring symbols at node $R_{j}, j=1,2$ or at the destination and its AMT nodes, owing to sampling or matched filtering (whatever pulse shaping is used).

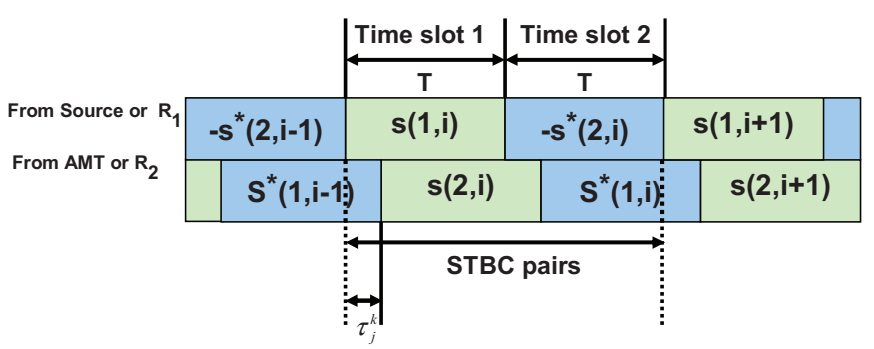

Fig. 3. Representation of the misalignment of the received signals at either the relay or destination nodes which induces ISI. 
In the first hop we assume that relay nodes $R_{j}, j=1,2$ are synchronized to the source node, while in the second hop the destination node and its AMT node are synchronized to $R_{1}$. The interference term is added to the received signals in both hops due to the asynchronism with the source AMT in the first hop and with $R_{2}$ in the second hop, note we assume integer sampling as all interference is modelled by single additive term, the received signal in both hops can be expressed as

$$
\begin{aligned}
r_{j}^{k}(t, i) & =h_{1 j}^{k}(0) S_{t 1}^{k}+h_{2 j}^{k}(0) S_{t 2}^{k}+I_{j}^{k}(t, i) \\
& +n_{j}^{k}(t, i), \text { for } j=1,2, k=1,2 \text { and } t=1,2(2)
\end{aligned}
$$

where

$$
\begin{gathered}
I_{j}^{k}(1, i)=h_{2 j}^{k}(-1) s^{*}(1, i-1) \\
I_{j}^{k}(2, i)=h_{2 j}^{k}(-1) s(2, i) .
\end{gathered}
$$

These quantities are vectorised for use in the following section as

and

$$
\begin{aligned}
\mathbf{r}_{H o p_{k}}(i) & =\left[r_{1}^{k}(1, i), r_{1}^{* k}(2, i), r_{2}^{k}(1, i), r_{2}^{* k}(2, i)\right]^{T} \\
\mathbf{n}_{H o p_{k}}(i) & =\left[n_{1}^{k}(1, i), n_{1}^{* k}(2, i), n_{2}^{k}(1, i), n_{2}^{* k}(2, i)\right]^{T}
\end{aligned}
$$

$$
\mathbf{I}_{H_{o p_{k}}(i)}=\left[I_{1}^{k}(1, i), I_{1}^{* k}(2, i), I_{2}^{k}(1, i), I_{2}^{* k}(2, i)\right]^{T} .
$$

where $n_{j}^{k}(t, i) \in C N\left(0, \sigma_{n}^{2}\right)$ is additive white Gaussian noise, $h_{1 j}^{k}(0)$ and $h_{2 j}^{k}(0)$ are the channel gains which are assumed constant within the block (note that $h_{1 j}^{k}(0)=h_{1 j}^{k}$ ), and $h_{2 j}^{k}(-1), k=1,2$ reflect the ISI from the previous symbols due to imperfect synchronization at the relay nodes in the first hop and at the destination node and its AMT node in the second hop; $h_{2 j}^{k}(-1)$ depends upon timing delay $\tau_{j}^{k}$ and the particular pulse shaping waveforms used and its relative strength will be expressed as a ratio as follows.

$$
\beta^{k}=\left|h_{2 j}^{k}(-1)\right|^{2} /\left|h_{2 j}^{k}(0)\right|^{2}
$$

where $\beta^{k}$ is defined to reflect the impact of time delay $\tau_{j}^{k}$ and pulse shaping waveforms. Similarly, inequality $\left|h_{2 j}^{k}(-1)\right|^{2}+\left|h_{2 j}^{k}(0)\right|^{2} \leq$ $\left|h_{2 j}^{k}\right|^{2}$ is used. When $\beta^{k}=0$, that means all nodes are perfectly synchronized to the received nodes and $\tau_{j}^{k}=0$. On the other hand, when $\beta^{k}=1$ (i.e. $0 \mathrm{~dB}$ ), that means, there is a time delay between the nodes at the received nodes and $\tau_{j}^{k}=0.5 T$. For fair comparison with a non-relay scheme, all terminal nodes transmit at $1 / 2$ power i.e. $\sigma_{r_{j}^{k}(t, i)}^{2}=\sigma_{s}^{2} / 2, j, t, k=1,2[16]$.

Phase two relaying: As shown in Fig. 2, the symbols detected at $R_{j}, j=1,2$ are re-encoded using the space time block coding technique and transmitted to the destination node and the AMT at the destination which is cooperating with the destination node to deliver good performance. The relay mode of decode and forward (DF) is used in this paper. We Denote the encoding data packet at $R_{j}$, $j=1,2$ corresponding to $\mathbf{s}(i)$ as $r_{j}^{1}(t, i)=\left[r_{j}^{1}(1, i), r_{j}^{1}(2, i)\right]^{T}$, $j=t=1,2$ as shown in equation (2). The codeword $S^{2}$, which is transmitted by $R_{1}$ and $R_{2}$ is therefore

$$
S^{2}=\left[\begin{array}{ll}
r_{1}^{1}(1, i) & r_{2}^{1}(1, i) \\
r_{1}^{1}(2, i) & r_{2}^{1}(2, i)
\end{array}\right]=\left[\begin{array}{cl}
s(1, i) & s(2, i) \\
-s^{*}(2, i) & s^{*}(1, i)
\end{array}\right]
$$

As shown in Fig. 3 and due to different propagation delays the transmitted signal $r_{j}^{1}(t, i)$ from relay nodes in the second hop will most likely arrive at the destination node and its AMT node at different time instants, as we mentioned in phase one. Therefore, the received signal at the destination and AMT at the destination can then also be represented as shown in equations (2), (3) and (4).

\section{CONVENTIONAL STBC DETECTION}

The conventional STBC detection can be carried out via the following standard two step procedure with assuming perfect channel state information (CSI) in both two hops. The detection vector of $\mathbf{g}_{\text {Hop }_{k}}(i)=\left[g_{H o p_{k}}(1, i), g_{H o p_{k}}(2, i)\right]^{T}, k=1,2$ with the received vector $\mathbf{r}_{\text {Hop }_{k}}(i)=\left[r_{1}^{k}(1, i), r_{1}^{* k}(2, i), r_{2}^{k}(1, i), r_{2}^{* k}(2, i)\right]^{T}$ can be calculated as follows

STEP 1: linear transform

$$
\begin{aligned}
\mathbf{g}_{\mathrm{Hop}_{k}}(i) & =\left[g_{\mathrm{Hop}_{k}}(1, i), g_{\mathrm{Hop}_{k}}(2, i)\right]^{T}=\mathbf{H}_{\mathrm{Hop}}^{\mathrm{H}} \mathbf{r}_{\mathrm{Hop}_{k}}(i) \\
& =\Delta_{\mathrm{Hop}_{k}} \mathbf{s}(i)+\mathbf{H}_{H o p_{k}}^{\mathrm{H}} \mathbf{I}_{H o p_{k}}(i) \\
& +\mathbf{H}_{\mathrm{Hop}_{k}}^{\mathrm{n}} \mathbf{n}_{\mathrm{Hop}_{k}}(i)
\end{aligned}
$$

Due to the Alamouti structure [1]

$$
\Delta_{H o p_{k}}=\mathbf{H}_{H o p_{k}}^{H} \mathbf{H}_{H o p_{k}}=\left[\begin{array}{ll}
\lambda_{H o p_{k}} & 0 \\
0 & \lambda_{H o p_{k}}
\end{array}\right]
$$

where

$$
\mathbf{H}_{H_{o p_{k}}}=\left[\begin{array}{rr}
h_{11}^{k}(0) & h_{21}^{k}(0) \\
h_{21}^{* k}(0) & -h_{11}^{* k}(0) \\
h_{12}^{k}(0) & h_{22}^{k}(0) \\
h_{22}^{* k}(0) & -h_{12}^{* k}(0)
\end{array}\right]
$$

and

$$
\lambda_{H_{o p_{k}}}=\left|h_{11}^{k}(0)\right|^{2}+\left|h_{21}^{k}(0)\right|^{2}+\left|h_{12}^{k}(0)\right|^{2}+\left|h_{22}^{k}(0)\right|^{2}
$$

STEP 2: The least squares (LS) method can be used to detect which symbols were actually transmitted.

$$
\hat{s}_{H o p_{k}}(t, i)=\arg \left\{\min _{s_{t} \in S}\left|g_{H o p_{k}}(t, i)-\lambda_{H o p_{k}} s_{t}\right|^{2}\right\}
$$

where $t, k=1,2$ and $S$ is the alphabet containing 8 symbols for 8-PSK.

The above procedure can suffer from significant detection error, because the $\mathbf{H}_{H o p_{k}}^{H} \mathbf{I}_{H_{o p}}(i)$ component in (8) will damage the orthogonality of the STBC. In the case of perfect synchronization when $h_{2 j}^{k}(-1)=0$, this procedure can however deliver good performance gain. 


\section{PARALLEL INTERFERENCE CANCELATION (PIC) DETECTION}

To overcome the impact of imperfect synchronization in both hops the interference component of $I_{j}^{k}(t, i)$ should be removed from (2) in both hops, assuming perfect channel information at both received nodes. Therefore, the principle of parallel interference cancelation is applied to remove the impact of imperfect synchronization of the interference component $I_{j}^{k}(t, i)$. Previously, PIC detection has been applied to a co-located STBC system [17]. Since $s^{*}(1, i-1)$ in (3) is already known in both hops if the detection process has been initialization properly, $I_{j}^{k}(t, i)$ can be removed during the initialization stage from both hops. The PIC iteration process can then be used to mitigate the imperfect value of $I_{j}^{k}(t, i)$ in (3) in both hops as shown in the following flowchart.

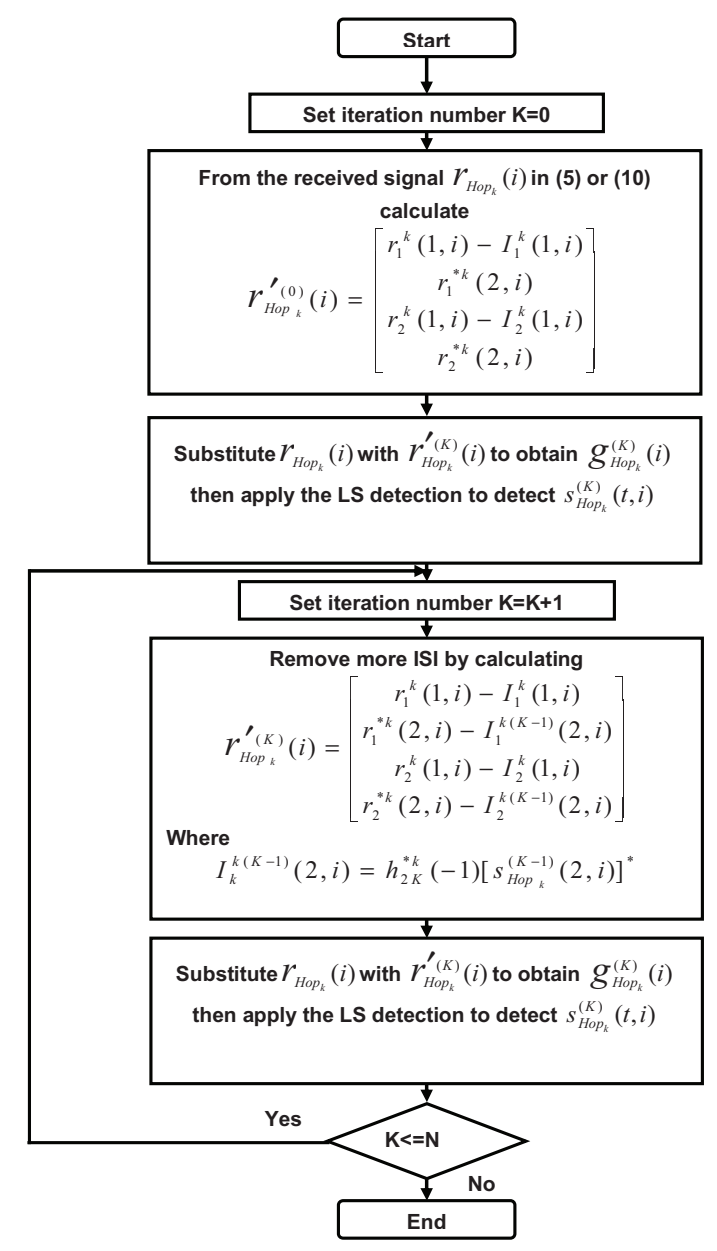

Fig. 4. The flowchart shows the PIC iteration process, which is used to mitigate the interference component at symbol level

The above algorithm will be maximum likelihood due to STBC structure, if the interference component $I_{j}^{k}(t, i)$ is removed. Most of the performance gain will be achieved in 2-3 iterations of the PIC process, which means the PIC detection is very effective in mitigating the error of synchronization as will be shown later in the simulation.

\section{SIMULATION RESULTS}

Simulation results of our proposed relaying solution under imperfect synchronization are given in this section. All simulations which have performed in this paper are uncoded. The BER performance against signal-to-noise ratio (SNR) was simulated by using 8-PSK gray mapping scheme. The SNR is defined as SNR $\sigma_{s}^{2} / \sigma_{n}^{2}$ and all nodes transmit at $1 / 2$ power. Fig. 5 compares the two stage direct transmission assisted cooperation [11] under perfect synchronization i.e. no time delay and our proposed solution under large time misalignments $\beta^{k}=0 d B$ and when PIC iteration is $\mathrm{K}=3$, it is clear that our scheme still provides an improved performance, for instance at BER of $10^{-2}$, our scheme provides a power saving of approximately $2 d B$ as compared with direct transmission assisted cooperation in [11]. Hence, the rest of the simulations do not consider direct transmission. In Figs. 6 and 7, the impact of the level of synchronization is shown by changing the value of $\beta^{k}$, Fig. 6 , shows the result of using the conventional detector with the D-STBC under different $\beta^{k}$ values. It can be observed that the conventional detector is not effective with synchronization error even under small time misalignments $\beta^{k}=-5 d B$ as shown in Fig. 6. On the other hand, the PIC scheme is very effective in mitigating synchronization error even at $\beta^{k}=0 d B$ as shown in Fig. 7 when the number of PIC iterations $\mathrm{K}=3$. Fig. 8 , illustrates the BER of PIC iterations for $\mathrm{K}=0,1,2,3$ and $\beta^{k}=-5 d B$. As shown in Fig 8 to achieve BER $10^{-3}$ approximately $17 d B$ SNR is necessary in the case of 2-stages perfect synchronization and in case of using PIC detection when $K=2,3$ and $\beta^{k}=-5 d B$, approximately $17.2 d B$ SNR is necessary. Therefore, the PIC scheme is very effective to mitigate the impact of imperfect synchronization, the second and third iteration deliver the performance gain, while the conventional detector dose not deliver the performances gain under imperfect synchronization.

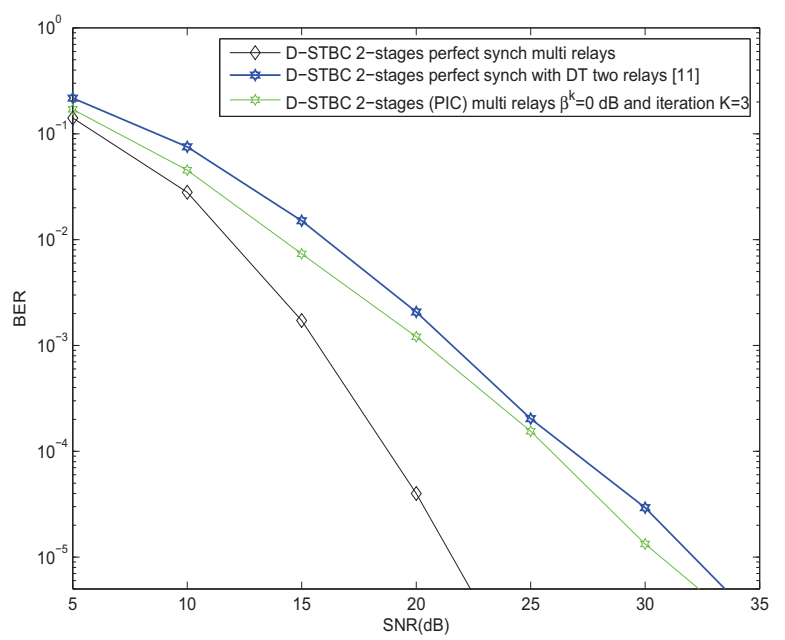

Fig. 5. End-to-end BER performance for 2-stage perfect synchronization with DT in [12] compared with our proposed solution when when $\beta^{k}=0 d B$ and $K=3$. 


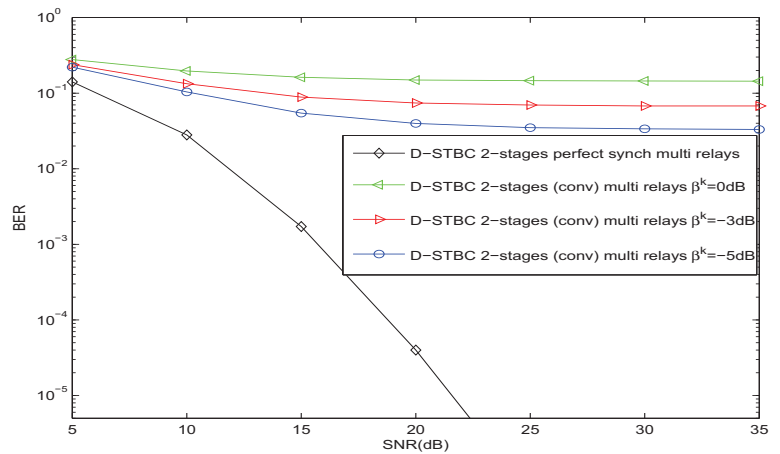

Fig. 6. End-to-end BER performance of conventional detector under different $\beta_{k}$ values.

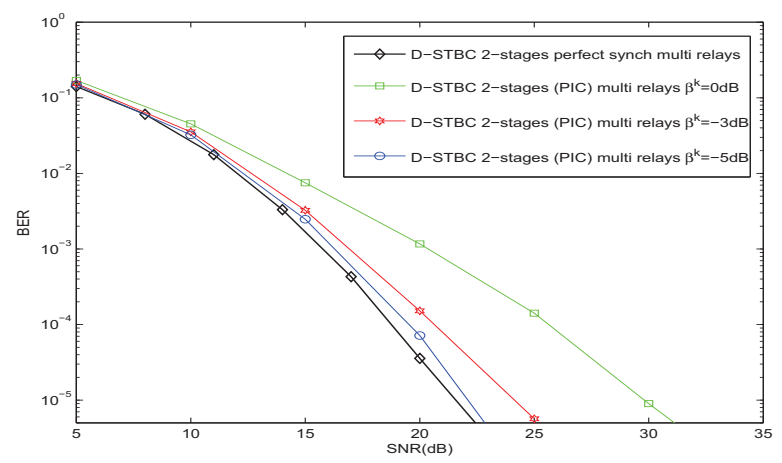

Fig. 7. End-to-end BER performance of PIC detector under different $\beta_{k}$ values and when the number of iterations $\mathrm{K}=3$.

\section{CONCLUSION}

In this paper, we have investigated the use of a parallel interference (PIC) technique in a cooperative communication systems. In particular, interference due to asynchronism within both hops is considered. We show the conventional D-STBC detector is very sensitive to synchronization error as compared with the PIC detector. Furthermore, the use of a multilevel cooperative multi-hop solution provides a significant improvement in networks as compared to the direct transmission assisted cooperation. Our solution thereby provides a relaying approach that is more robust to both small and large scale fading, and has the flexibility to incorporate interference cancellation.

\section{REFERENCES}

[1] A.M. Alamouti, "A simple transmit diversity techniques for wireles communications," IEEE J SAC, vol.16, no.8, pp.1451-1458, October 1998.

[2] J. Laneman, D. Tse, and G. Wornell, "Cooperative diversity in wire less networks: efficient protocols and outage behaviour," IEEE Trans. Inform. Theory, vol. 50, no. 12, pp. 3062-3080, December 2004.

[3] M.Dohler, Virtual Antenna Arrays, PhD Thesis, University of London. Nov. 2003.

[4] S. Yiu, R. Schober and L. Lampe, "Distributed space-time block cod-

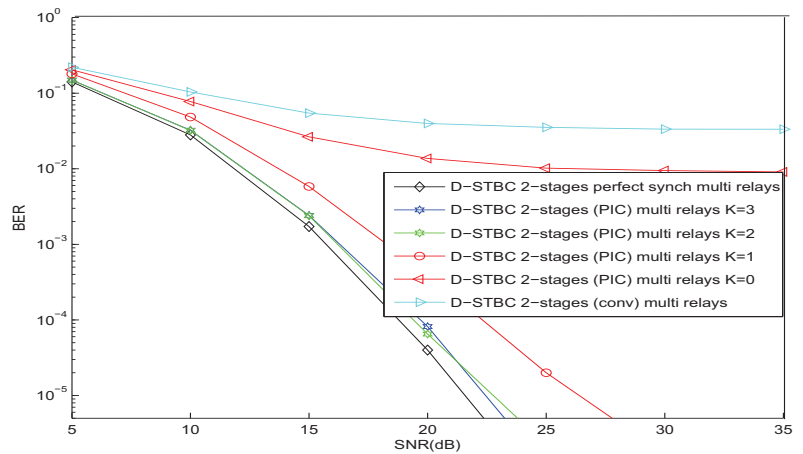

Fig. 8. End-to-end BER performance of PIC detector when $\beta_{k}=-5 d B$ with different number of iterations $\mathrm{K}$.

ing," IEEE Trans. on Communications, Vol. 54, No. 7, pp.1195-2006, July 2006.

[5] J. Mietzner and P. A. Hoeher, "Distributed space-time codes for cooperative wireless networks in the presence of different propagation delays and path Losses," IEEE Sensor Array and Multichannel Signal Processing Workshop, pp.264 - 268, July, 2004.

[6] S.K. Kassim, M. Hayes, N.M. Eltayeb and J.A. Chambers, "Exploitation of quasi-orthogonal space time block code in virtual antenna arrays: Part II Monte Carlo-based throughput evaluation," 67th IEEE Vehicular Technology Conf. (VTC), Vol. 5, pp.342 - 346, May 2008.

[7] N.M. Eltayeb, S.K. Kassim, and J. A. Chambers, "Two-hop amplifyand-forward scheme for extended orthogonal space time coding in cooperative networks," IEEE Inter. Symposium on Information Theory and its Applications (ISITA), pp.1 - 5, Dec. 2008.

[8] X. Li , "Space time coded multi-transmission among distributed transmitter without perfect syncronisation," IEEE Signal Processing Lett. Vol. 11, No. 12, pp. 948 - 951, Dec. 2004.

[9] X. Li, F.Ng, J-T. Hwu and M. Chen, "Channel equalization for STBC-encoded cooperative transmission with asynchronous transmitter," 39th IEEE Asilomar Conference on Signals, Systems, and Computers, pp.457 - 461, Oct. 2005.

[10] D. Divsalar, M.K. Simon and D. Raphaeli , "Improved parallel interefence cancellation for CDMA," IEEE Trans. on Communications, Vol. 46, No. 2, pp.258 - 268, Feb. 1998.

[11] F.-C. Zheng, A.G. Burr and S.Olafsson, "PIC detector for distributed space-time block coding under imperfect synchronisation," IET Electronics Letters, vol. 43. no. 10, pp. 580-581, May 2007.

[12] T.M. Cover, and J.A. Thomas, "Element of information theory," New York: Wiley, 1991.

[13] L. Le and E. Hossain, "Multihop Cellular Networks: Potential Gains, Research Challenges, and a Resource Allocation Framework,'IEEE Communication Magazine, Vol.45, pp.66 - 73, Sept. 2007.

[14] R. Morrow, Bluetooth Operation and Use, McGraw-Hill, 2002.

[15] S. Wei, D. Goeckel, and M. Valenti, "Asynchronous Cooperative Diversity," IEEE Trans. on Wireless Communication, vol. 5, no. 6, pp. 1547-1557, June 2006

[16] X. Li, F. Ng, J. Hwu, and M. Chen, "Channel equalization for STBCencoded cooperative transmissions with asynchronous transmitters," Proc. of the 39th Asilomar conf. on Signals Systems and Computer, pp.457-461, October 2005.

[17] F. Zheng, A. Burr, and S. Olafsson, "Signal detection for orthogonal space time block coding over time selective fading channel: a PIC approach for the $\mathcal{G}_{i}$ system" IEEE Trans. Communication, vol. 53,no. 6, pp. 969-972, June 1998. 